\title{
Electrochemical Capacitance DNA Sensing at Hairpin-Modified Au Electrodes
}

\author{
Joel Rivera-Gandía, Maria del Mar Maldonado, Yarimar De La Torre-Meléndez, \\ Edwin O. Ortiz-Quiles, Nella M. Vargas-Barbosa, and Carlos R. Cabrera
}

\begin{abstract}
Department of Chemistry and Center for Advanced Nanoscale Materials, University of Puerto Rico, Río Piedras Campus, P.O. Box 23346, San Juan, PR 00931-3346, USA
\end{abstract}

Correspondence should be addressed to Carlos R. Cabrera, carlos.cabrera2@upr.edu

Received 1 September 2010; Revised 7 April 2011; Accepted 23 June 2011

Academic Editor: Jiri Homola

Copyright ( $\odot 2011$ Joel Rivera-Gandía et al. This is an open access article distributed under the Creative Commons Attribution License, which permits unrestricted use, distribution, and reproduction in any medium, provided the original work is properly cited.

\begin{abstract}
An interfacial capacitance measurement electrochemical technique has been used for the sensing of self-assembled DNA hairpin probes (M. tuberculosis and B. anthracis) attached to Au electrodes. The double-layer capacitance $\left(C_{\mathrm{dl}}\right)$ was determined with electrochemical perturbations from $0.2 \mathrm{~V}$ to $0.5 \mathrm{~V}$ versus $\mathrm{Ag} / \mathrm{AgCl}$ at a $\mathrm{Au} / \mathrm{M}$. tuberculosis DNA hairpin probe at surface coverage $\mathrm{Au}$ electrodes. The capacitance study was done at $\mathrm{pH}$, which was necessary to maintain the M. tuberculosis and B. anthracis DNA probes closed during the electrochemical perturbation. Detailed experimental analysis carried out by repetitively switching the electrochemical potential between 0.2 and $0.5 \mathrm{~V}$ (versus $\mathrm{Ag} / \mathrm{AgCl}$ ) strongly supports the use of capacitance measurements as a tool to detect the hybridization of DNA targets. A large change in the capacitance deference between 0.2 and $0.5 \mathrm{~V}$ was observed in the DNA hybridization process. Therefore, no fluorophores or secondary transducers were necessary to sense a DNA target for both DNA hairpins.
\end{abstract}

\section{Introduction}

The behavior of DNA attached onto metallic and nonmetallic surfaces via self-assembly with various chemistries (e.g., Au-S) may have applications in biomedical devices. For example, single-stranded DNA (ssDNA) self-assembled on a metallic interface such as gold $[1,2]$ or on nonmetals such as carbon nanotubes [3] and diamond [4-6] has potential use in DNA microarrays [7]. In addition, detection of DNA hybridization has been possible with techniques using different types of reporting, including fluorescence [8-11], chronocoulometry [12-14], surface plasmon resonance (SPR) [15, 16], colloidal labeling [17-19], and polarization modulation infrared reflection absorption spectroscopy [20]. Electroactive molecules can also be used to monitor the electron transfer mechanism during the hybridization process [21]. Here, we present a nonfaradaic electrochemical method based on capacitive measurement to sense DNA hairpin modification and hybridization.
A nucleic acid probe has been developed to recognize specific DNA targets in solution [22]. These probes, called molecular beacons, are DNA hairpins with a fluorophorequencher pair, which is completely unable to fluoresce when the two components are in close proximity (i.e., closed molecular beacon). When the molecular beacon spontaneously changes its conformation (like during hybridization), the fluorophore attached to one end of the molecule is no longer quenched as the quencher moves away. The capacity of this DNA hairpin has shown to discriminate between alleles with high specificity when compared with linear DNA probes $[23,24]$. Discrimination also has been demonstrated among four single-nucleotide variants when DNA amplification and DNA detection are carried at the same time in solution [25]. The thermodynamics of the DNA hairpin and its enhanced specificity in detection have been studied [26]. In solution in the presence of the target, the hairpin exists mainly in three states: (1) hybridized to the target DNA, (2) closed hairpin, and (3) as a random coil (i.e., as in nonhybridized 
open hairpin). The structural constrains of these three conformational states lead to the enhanced specificity. Hairpin sequence and structure may also play an influence on the binding characteristics $[27,28]$. Recently, DNA sensors with hairpin probes have been presented by Liu et al. [29] and Song et al. [30].

The mechanism used to report on hybridization must be useful under the conditions affecting the hybridization process itself (i.e., secondary structure, ionic strength, and sequence). For example, a reporting technique based on fluorescence requires a fluorophore with reasonable fluorescence quantum yield. This is affected by solvent polarity, ionic strength, and proximity to a secondary energy recipient. Electrochemical detection also requires the following as a minimum: good diffusion to the electrode, good affinity with the probe, and conformation between probe and target [8-11].

Currently, two main techniques show the most potential to report the hybridization of probe-target DNA under conditions where the end product is not affected by the force to meet with the reporter requirements: surface plasmon resonance (SPR) $[15,16]$ and electrochemical impedance spectroscopy (EIS) [31].

Previously, faradaic EIS has been used to detect the hybridization of target DNA strands with DNA probes tethered onto diamond and silicon surfaces $[6,32]$. Nonfaradaic electrochemical detection of proteins interactions has been demonstrated by Jacquot et al. using chemoreceptive neuron metal-oxide-silicon (CvMOS) [33]. Willner's group has worked on impedance measurements for DNA and other biomolecule sensing as well $[34,35]$. Recently, we demonstrated that nonfaradaic EIS could be used to study ssDNA conformational changes under potential perturbations at the electrode-liquid interface. We were able to measure precisely the double-layer capacitance behavior of DNA strands attached to gold surfaces. The electric potential perturbation induced conformational changes in the ssDNA monolayer, which were monitored by following the changes in $C_{\mathrm{dl}}$ by EIS. This technique allowed us to explore DNA/surface interfacial phenomena in detail.

In this study, we propose a new approach to characterize the electrode-electrolyte interface through non-faradic capacitive measurements by switching the applied electrochemical potential between the potential of zero charge $(\mathrm{PZC})$ of the monolayer $(0.2 \mathrm{~V})$ and a positive potential $(0.5 \mathrm{~V})$. The capacitance at the potential of zero charge (PZC) is used as a reference state in which the capacitance at a higher potential is compared. To demonstrate the advantage of this methodology, we report the hybridization of two DNA hairpins self-assembled monolayers (HS$\left(\mathrm{CH}_{2}\right)_{6}$-GCGAGGAACGGCTGATGACCAAACTCTCGC, M. tuberculosis hairpin) and (HS- $\left(\mathrm{CH}_{2}\right)_{6}$-CCGACGAGGGTTGTCAGAGGATGCGTCGG, B. anthracis hairpin). Both hybridization events detection by non-faradic ESI used a frequency response analyzer (FRA).

\section{Experimental Section}

2.1. Materials. Trizma-Base, $\mathrm{NaH}_{2} \mathrm{PO}_{4}$, and $\mathrm{MgSO}_{4}$ from Sigma-Aldrich were used as received to prepare, respectively, the $200 \mathrm{mM}, 100 \mathrm{mM}$, and $5 \mathrm{mM}$ aqueous buffer solutions at a $\mathrm{pH}$ of 6.15. Hairpin probes HS- $\left(\mathrm{CH}_{2}\right)_{6}$-GCGAGGAACGGCTGATGACCAAACTCTCGC (M. tuberculosis hairpin (MTHP)) and HS- $\left(\mathrm{CH}_{2}\right)_{6}$-CCGACGAGGGTTGTCAGAGGATGCGTCGG (B. anthracis hairpin (BAHP)) and their respective targets CGCTCCTTGCCGACTACTGGTTTGAGAGCG (M. tuberculosis hairpin target (MTHPT) and GGCTGCTCCCAACAGTCTCCTACGCAGCC (B. anthracis hairpin target (BAHPT) were obtained from Synthegen, LLC. Upon receipt, the $M$. tuberculosis and B. anthracis hairpins samples were diluted with $1 \mathrm{~mL}$ of Nanopure water (Barnstead Nanopure; $18.0 \mathrm{M} \Omega-\mathrm{cm}$ ). Aliquots were transferred to different unit containers and stored at $4^{\circ} \mathrm{C}$ until use. Since the samples were received in disulfide form, before use, each aliquot was deprotected by diluting it with $1.5 \mathrm{~mL}$ of dithiothreitol (DTT) $0.4 \mathrm{M}$ solution in $0.17 \mathrm{M}$ phosphate buffer ( $\mathrm{pH} 8.0)$ and left overnight at room temperature. The aqueous fraction of the deprotected product was separated and desalted by running through a size-exclusion NAP10 column (Amersham Biosciences) preequilibrated with $0.01 \mathrm{M}$ sodium phosphate buffer ( $\mathrm{pH}$ 6.8). The deprotected and desalted sample was diluted with the Trizma-Na-Mg buffer $\mathrm{pH}=6.15$, to obtain a $2 \mu \mathrm{M}$ solution of $M$. tuberculosis and $B$. anthracis hairpins.

2.2. Cyclic Voltammetry and Electrochemical Impedance Spectroscopy (EIS). A potentiostat (Eco Chemie Autolab PGSTAT302) was used to perform the cyclic voltammetry for electrode characterization. The electrochemical impedance spectroscopy was with the potentiostat and a frequency response analyzer (FRA2) module. The frequency range used for EIS ranged from $100 \mathrm{kHz}$ to $100 \mathrm{MHz}$, with AC amplitude of $10.0 \mathrm{mV} \mathrm{rms.}$

2.3. Au Electrode Preparation. A polished polycrystalline gold electrode $\left(0.02 \mathrm{~cm}^{2}\right.$, BioAnalytical Systems) was used as the working substrate for all experiments. The gold surface was polished to optical smoothness with successive Carbimet papers and $\mathrm{Al}_{2} \mathrm{O}_{3}$ powder (Alpha Micropolish II deagglomerated Alumina; $5 \mu \mathrm{m}, 0.3 \mu \mathrm{m}$, and $0.05 \mu \mathrm{m}$ ) from Buehler, Lake Bluff, Ill, USA. The electrode was rinsed with Nanopure water and sonicated for 20 minutes in ethanol (99.9\%). The electrode then was electrochemically etched in $0.5 \mathrm{M}$ sulfuric acid for about 20 minutes or until the electrode exhibited a distinctive decrease in the oxygen reduction peak. Upon etching, additional oxidation and reduction peaks appeared, as if etched gold byproduct has clung to the surface. To eliminate those peaks, the electrode was sonicated for about 20 minutes in ethanol and was characterized by cyclic voltammetry in $0.5 \mathrm{M}$ sulfuric acid to determine the surface crystalline state and roughness factor. The roughness factor for this work ranged from 1.2 to 1.5 .

2.4. M. tuberculosis Hairpin (MTHP) Self-Assembled Monolayer. The prepared Au electrode was immersed in the MTHP sample diluted in Trizma-Na-Mg buffer at two different immobilization times: $2 \mathrm{hrs}$ for the low surface coverage (Au/MTHP-L) and $16 \mathrm{hrs}$ for high surface coverage 
$(\mathrm{Au} / \mathrm{MTHP}-\mathrm{H})$. Then, the electrode was rinsed with the Trizma-Na-Mg buffer and placed in the electrochemical cell for the EIS measurements.

2.5. B. anthracis Hairpin (BAHP) Self-Assembled Monolayer. The prepared $\mathrm{Au}$ electrode was immersed in the BAHP sample diluted in Trizma-Na-Mg buffer for $16 \mathrm{hrs}$ for high surface coverage (Au/BAHP-H). Then, the electrode was rinsed with the Trizma-Na-Mg buffer and placed in the electrochemical cell for the EIS measurements.

2.6. Hybridization to M. tuberculosis (MTHP) Complementary Target. Hybridization was performed ex situ, by immersing the modified electrode with the MTHP into a $9.5 \mu \mathrm{M}$ solution in Trizma-Na-Mg buffer for 1 hour. Then, the electrode was rinsed with the Trizma-Na-Mg buffer and placed back in the electrochemical cell for the EIS measurements.

\section{Results and Discussion}

DNA hybridization has been monitored using different reporting techniques and devices $[8,9]$. However, genetic testing requires the development of easy, fast, and inexpensive devices to report the hybridization event. Electrochemically based biosensors for DNA hybridization need to meet these requirements, and pose high specificity. Electrochemical transducers have been shown to detect the hybridization event [36-38]. The high sensitivity of electrochemical transducing devices and their potential for minimal power requirements among other advantages make them suitable for large-scale production for this use.

Among the transducing techniques reported are the uses of redox indicator or from changes in DNA capacitance/ conductivity before and after the hybridization event. Most of the capacitive measurements have been performed in a faradaic environment with a redox indicator, including a novel use of an ionchannel with a ferrocyanide redox marker [39]. On the other hand, electrochemical impedance spectroscopy (EIS) reliably measures the interfacial capacitance at fixed potentials, free from background currents commonly observed in cyclic voltammetry [40]. EIS allowed us to make high-precision measurements in order to relate the resistance and capacitance values to interfacial phenomena and the double-layer structure. The use of insulating monolayers minimized the effect of the double layer attributed to $\mathrm{Au}$ electrode-electrolytes own capacitance. In the GouyChapman-Stern model, the effect of the coated electrode double layer is attributed to two regions: the compact layer $\left(C_{\text {Helmholtz }}\right)$, which is assumed to be an ideal capacitor, and the diffuse double layer $\left(C_{\text {Gouy-Chapman }}\right)$.

In our experimental setup, the capacitance of the compact (Helmholtz) layer was replaced with the capacitance of the MTHP or BAHP monolayer. Therefore, the electrode capacitance per $\mathrm{cm}^{2}, C_{\mathrm{dl}}$, was then represented by

$$
\frac{1}{C_{\mathrm{dl}}}=\frac{1}{C_{\text {Hairpin }}}+\frac{1}{C_{\text {Gouy-Chapman }}},
$$

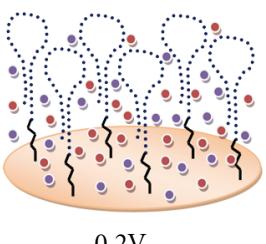

$0.2 \mathrm{~V}$

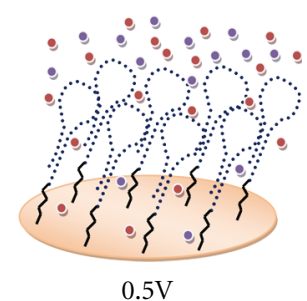

FIgURE 1: Schematic representation of Au/Hairpin monolayer at high surface coverage at the PZC $(0.2 \mathrm{~V})$ and at $0.5 \mathrm{~V}$. Cations are represented by blue dots, and anions by red dots.

where $C_{\text {Hairpin }}$ is the capacitance of the MTHP or BAHP monolayer per $\mathrm{cm}^{2}$ and $C_{\text {Gouy-Chapman }}$ is the capacitance contribution from the diffuse layer per $\mathrm{cm}^{2}$.

We have reported on the effectiveness of the electrochemical switching on the conformation of specifically absorbed ssDNA [31]. The difference in conformation of single-stranded DNA (ssDNA) at the PZC $(0.2 \mathrm{~V})$ and at $0.5 \mathrm{~V}$ was demonstrated by the difference in the resulting capacitance. The inherent flexibility of the ssDNA and the response of the phosphate backbone to the applied potential at $0.5 \mathrm{~V}$ make the ssDNA pulled closer to the electrode surface. At the PZC $(0.2 \mathrm{~V})$, the ssDNA will return back to its initial conformation. This electrochemically induced conformational change can also be explained by the change in ion composition at each applied potential. During the change from the PZC $(0.2 \mathrm{~V})$ to $0.5 \mathrm{~V}$, the cations are pushed away, while the anions are attracted to the electrode to keep the charge balance.

This thermodynamics of the double layer are well explained by the Gibbs adsorption isotherm. The interfacial zone can be described as the region in which intermolecular forces can be perturbed by the electrochemical potential inducing changes on the properties of a surface. Therefore, we can choose a reference interfacial state where the intermolecular forces are unperturbed, at equilibrium. This reference interfacial state is attained at the potential of zero charge (PZC), where the charge at the Au electrode and interface is zero.

From this reference state, the electrode surface can be perturbed to either negative or positive potentials to create charge excess that will induce a change in the interface characteristics to store charge in response to the perturbation

$$
C_{\mathrm{dl}}=\frac{\partial \sigma^{M}}{\partial E} .
$$

In our experiments with hairpins monolayer, we found different capacitive behaviors as compared to the ssDNA monolayer [31]. The capacitance for ssDNA monolayer was lower at PZC $(0.2 \mathrm{~V})$, in contrast to the observed capacitance at $0.2 \mathrm{~V}$ for the hairpins. As presented in Figure 1, this difference in capacitive current could be explained by the greater blocking capacity of the compact hairpin monolayer at $0.5 \mathrm{~V}$ than at $0.2 \mathrm{~V}$, preventing ions from approaching the surface at the higher voltage. At higher voltage positive to the PZC, the interfacial characteristics and charge 


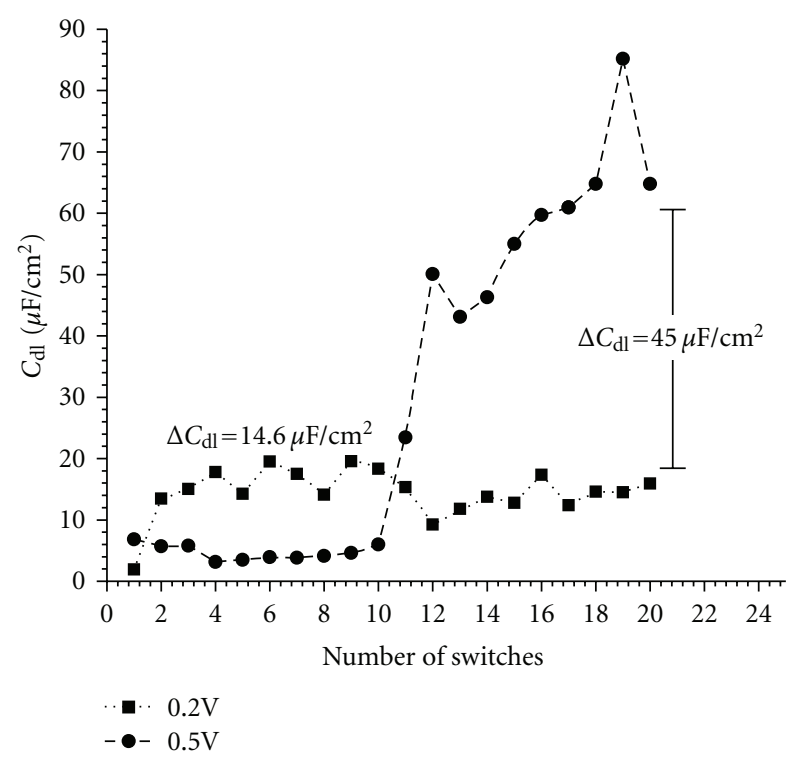

Figure 2: M. tuberculosis hairpin hybridization detected by nonfaradaic charging electrochemical impedance spectroscopy. The hybridization process was done at potential switch number 10 .

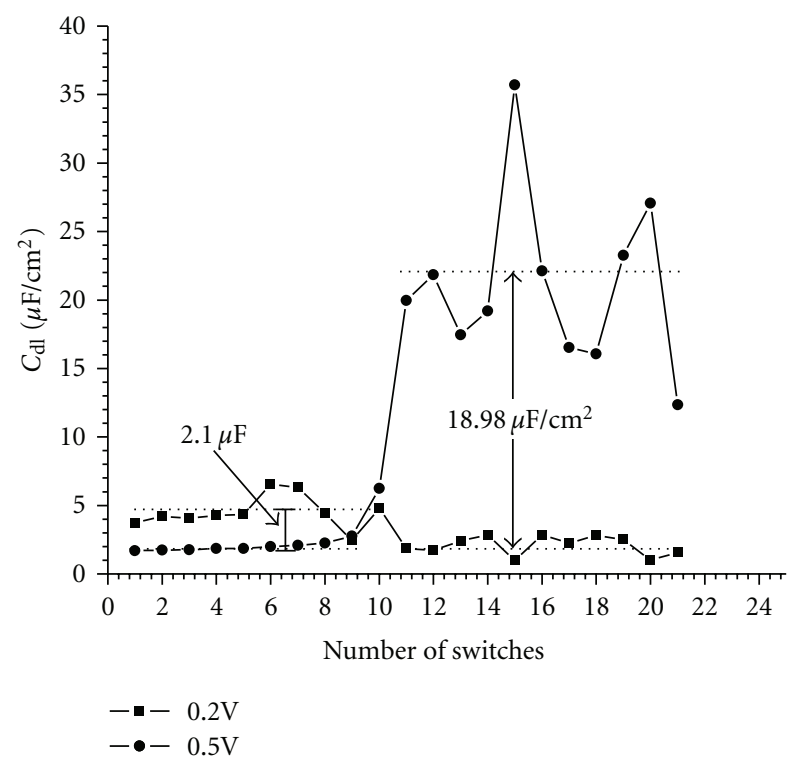

FIGURE 3: B. anthracis hairpin hybridization detected by nonfaradaic charging electrochemical impedance spectroscopy. The hybridization process was done at potential switch number 10 .

change in response to the applied potential. The hairpin monolayer responds to the perturbation by the attraction of the phosphate backbone to the surface tilting the hairpin monolayer. Barton et al. have described this through AFM studies in which a polyanionic DNA can be either in upright position for repelling negative potentials or flat on surface for attractive positive potentials [41]. The monolayer formed from closed $M$. tuberculosis hairpin shows a difference between $0.2 \mathrm{~V}$ and $0.5 \mathrm{~V}$ of $14.6 \mu \mathrm{F} / \mathrm{cm}^{2}$ for the first 10 switches in Figure 2. This difference in capacitance indicates the formation of a compact monolayer with little response to electrochemical perturbation due to the closed hairpin.

After exposing the $M$. tuberculosis to its complementary strand for $1 \mathrm{hr}$, the capacitance difference is expected to increase due to the opening of the hairpin stem upon hybridization. The result presented in Figure 2 confirms it with a 3 -fold increase in capacitance difference to $45 \mu \mathrm{F} / \mathrm{cm}^{2}$. This increase upon hybridization is in agreement with a similar response reported after hybridization by relative fluorescent intensity using a fiber-optic evanescent wave DNA biosensor with molecular beacons probes [42].

To explore the opportunities of this nonfaradaic impedance, a second experiment was conducted. For this, a B. anthracis hairpin sample was prepared and tested. The interfacial characteristics of the hairpin monolayer show a similar profile. As seen in Figure 3, the capacitance difference between $0.2 \mathrm{~V}$ and $0.5 \mathrm{~V}$ for the closed $B$. snthracis hairpin $\left(2.1 \mu \mathrm{F} / \mathrm{cm}^{2}\right)$ is smaller in comparison to the closed state for the M. tuberculosis hairpin. This difference is attributed to the actual surface coverage for both hairpins, being the $B$. anthracis with the more compacted monolayer. Upon its hybridization with its target complementary strand, the $B$. anthracis hairpin opens, and the interfacial capacitance increases to $18.98 \mu \mathrm{F} / \mathrm{cm}^{2}$, which represents approximately 9 -fold increase from its closed state. The higher response is also in agreement with a higher surface coverage by the $B$. anthracis hairpin. The higher the surface coverage, the higher the capacitive response upon hybridization.

Though we used a reference state (PZC) for comparison, the capacitance increase presented at $0.5 \mathrm{~V}$ prior and after hybridization shows a 10 -fold for both hairpins. This is in agreement with the results obtained with molecular beacons and flow cytometry used to detect E. coli at different time periods [41].

\section{Conclusions}

We have reported the capacitance profile for tethered hairpins on gold surface. The differences in capacitance observed for closed hairpins can be used to characterize the nature of the surface coverage. For instance, each particular hairpin sequence will have a different conformation of its closed state affecting the actual surface coverage and subsequently the capacitance difference. In the same manner, the capacitance difference response upon hybridization will be affected by its initial surface coverage. Here, we presented the use of the capacitance at the PZC as a reference to demonstrate the hybridization of both DNA hairpins (M. tuberculosis and B. anthracis) on gold surface.

Based on previous work [31], we found that hairpins monolayer response upon switching the potential applied to the electrode surface shows a capacitance at $0.5 \mathrm{~V}$ below the capacitance at PZC. This is attributed to the higher blocking effect of the closed hairpin due to its tilt conformation upon electrochemical perturbation. At PZC, the closed hairpin allows the ions to approach the surface by diffusing through the hairpins [22]. 
Finally, M. tuberculosis and B. anthracis complementary strand were detected by a nonfaradaic electrochemical impedance spectroscopy method, following the changes in double-layer capacitive current from its initial compact to a looser-hybridized state. These findings demonstrate how differential capacitive measurements can be used as a transducer method in DNA sensing. Moreover, we have presented a nonfaradaic (nonredox) electrochemical base DNA detection method. Hairpins have been shown to have the potential to detect complementary sequence in complex samples (i.e., blood clinical samples) [31]. Therefore, the creation of a DNA hairpin-gold array is possible to detect DNA hybridization by monitoring the capacitance of the hairpin-gold monolayer through nonfaradaic impedance spectroscopy.

\section{Acknowledgments}

This project was partially funded by NASA-URC Grant nos. NCC3-1034 and NNX08BA48A and by P20 RR-16470 IDeA (Networks of Biomedical Research Excellence, INBRE). A NASA-GSRP Fellowship (NNA-05CM-02H) supported J. Rivera-Gandia.

\section{References}

[1] T. M. Herne and M. J. Tarlov, "Characterization of DNA probes immobilized on gold surfaces," Journal of the American Chemical Society, vol. 119, no. 38, pp. 8916-8920, 1997.

[2] S. O. Kelley, J. K. Barton, N. M. Jackson et al., "Orienting DNA helices on gold using applied electric fields," Langmuir, vol. 14, no. 24, pp. 6781-6784, 1998.

[3] C. S. Lee, S. E. Baker, M. S. Marcus, W. Yang, M. A. Eriksson, and R. J. Hamers, "Electrically addressable biomolecular functionalization of carbon nanotube and carbon nanofiber electrodes," Nano Letters, vol. 4, no. 9, pp. 1713-1716, 2004.

[4] W. Yang, O. Auciello, J. E. Butler et al., "DNA-modified nanocrystalline diamond thin-films as stable, biologically active substrates," Nature Materials, vol. 1, no. 4, pp. 253-257, 2002.

[5] T. Knickerbocker, T. Strother, M. P. Schwartz et al., "DNAmodified diamond surfaces," Langmuir, vol. 19, no. 6, pp. 1938-1942, 2003.

[6] W. Yang, J. E. Butler, J. N. Russell Jr., and R. J. Hamers, "Interfacial electrical properties of DNA-modified diamond thin films: intrinsic response and hybridization-induced field effects," Langmuir, vol. 20, no. 16, pp. 6778-6787, 2004.

[7] S. P. A. Fodor, "Massively parallel genomics," Science, vol. 277, no. 5324, pp. 393-395, 1997.

[8] S. P. A. Fodor, J. L. Read, M. C. Pirrung, L. Stryer, A. T. Lu, and D. Solas, "Light-directed, spatially addressable parallel chemical synthesis," Science, vol. 251, no. 4995, pp. 767-773, 1991.

[9] D. J. Lockhart and E. A. Winzeler, "Genomics, gene expression and DNA arrays,” Nature, vol. 405, no. 6788, pp. 827-836, 2000.

[10] M. L. Bulyk, E. Gentalen, D. J. Lockhart, and G. M. Church, "Quantifying DNA-protein interactions by double-stranded DNA arrays," Nature Biotechnology, vol. 17, no. 6, pp. 573-577, 1999.

[11] M. Chee, R. Yang, E. Hubbell et al., "Accessing genetic information with high-density DNA arrays," Science, vol. 274, no. 5287, pp. 610-614, 1996.
[12] M. B. Esch, L. E. Locascio, M. J. Tarlov, and R. A. Durst, "Detection of viable Cryptosporidium parvum using DNAmodified liposomes in a microfluidic chip," Analytical Chemistry, vol. 73, no. 13, pp. 2952-2958, 2001.

[13] A. B. Steel, T. M. Herne, and M. J. Tarlov, "Electrochemical quantitation of DNA immobilized on gold," Analytical Chemistry, vol. 70, no. 22, pp. 4670-4677, 1998.

[14] A. B. Steel, T. M. Herne, and M. J. Tarlov, "Electrostatic interactions of redox cations with surface-immobilized and solution DNA," Bioconjugate Chemistry, vol. 10, no. 3, pp. 419423, 1999.

[15] L. He, M. D. Musick, S. R. Nicewarner et al., "Colloidal Auenhanced surface plasmon resonance for ultrasensitive detection of DNA hybridization," Journal of the American Chemical Society, vol. 122, no. 38, pp. 9071-9077, 2000.

[16] J. M. Brockman, A. G. Frutos, and R. M. Corn, "A multistep chemical modification procedure to create dna arrays on gold surfaces for the study of protein-DNA interactions with surface plasmon resonance imaging," Journal of the American Chemical Society, vol. 121, no. 35, pp. 8044-8051, 1999.

[17] M. L. Sauthier, R. Lloyd Carroll, C. B. Gorman, and S. Franzen, "Nanoparticle layers assembled through DNA hybridization: characterization and optimization," Langmuir, vol. 18, no. 5, pp. 1825-1830, 2002.

[18] Y. Cao, R. Jin, and C. A. Mirkin, "DNA-modified coreshell Ag/Au nanoparticles," Journal of the American Chemical Society, vol. 123, no. 32, pp. 7961-7962, 2001.

[19] L. M. Demers, C. A. Mirkin, R. C. Mucic et al., "A fluorescencebased method for determining the surface coverage and hybridization efficiency of thiol-capped oligonucleotides bound to gold thin films and nanoparticles," Analytical Chemistry, vol. 72, no. 22, pp. 5535-5541, 2000.

[20] S. H. Brewer, S. J. Anthireya, S. E. Lappi, D. L. Drapcho, and S. Franzen, "Detection of DNA hybridization on gold surfaces by polarization modulation infrared reflection absorption spectroscopy," Langmuir, vol. 18, no. 11, pp. 4460-4464, 2002.

[21] C. Fan, K. W. Plaxco, and A. J. Heeger, "Electrochemical interrogation of conformational changes as a reagentless method for the sequence-specific detection of DNA," Proceedings of the National Academy of Sciences of the United States of America, vol. 100, no. 16, pp. 9134-9137, 2003.

[22] S. Tyagi and F. R. Kramer, "Molecular beacons: probes that fluoresce upon hybridization," Nature Biotechnology, vol. 14, no. 3, pp. 303-308, 1996.

[23] S. Tyagi, D. P. Bratu, and F. R. Kramer, "Multicolor molecular beacons for allele discrimination," Nature Biotechnology, vol. 16, no. 1, pp. 49-53, 1998.

[24] L. G. Kostrikis, S. Tyagi, M. M. Mhlanga, D. D. Ho, and F. R. Kramer, "Spectral genotyping of human alleles," Science, vol. 279, no. 5354, pp. 1228-1229, 1998.

[25] S. A. E. Marras, F. Russell Kramer, and S. Tyagi, "Multiplex detection of single-nucleotide variations using molecular beacons," Genetic Analysis_Biomolecular Engineering, vol. 14, no. 5-6, pp. 151-156, 1999.

[26] G. Bonnet, S. Tyagi, A. Libchaber, and F. R. Kramer, "Thermodynamic basis of the enhanced specificity of structured DNA probes," Proceedings of the National Academy of Sciences of the United States of America, vol. 96, no. 11, pp. 6171-6176, 1999.

[27] R. Bar-Ziv and A. Libchaber, "Effects of DNA sequence and structure on binding of RecA to single-stranded DNA," Proceedings of the National Academy of Sciences of the United States of America, vol. 98, no. 16, pp. 9068-9073, 2001. 
[28] Y. Gao, L. K. Wolf, and R. M. Georgiadis, "Secondary structure effects on DNA hybridization kinetics: a solution versus surface comparison," Nucleic Acids Research, vol. 34, no. 11, pp. 3370-3377, 2006.

[29] G. Liu, Y. Wan, V. Gau et al., "An enzyme-based E-DNA sensor for sequence-specific detection of femtomolar DNA targets," Journal of the American Chemical Society, vol. 130, no. 21, pp. 6820-6825, 2008.

[30] S. Song, Z. Liang, J. Zhang, L. Wang, G. Li, and C. Fan, "Goldnanoparticle-based multicolor nanobeacons for sequencespecific DNA analysis," Angewandte Chemie-International Edition, vol. 48, no. 46, pp. 8670-8674, 2009.

[31] J. Rivera-Gandía and C. R. Cabrera, "Self-assembled monolayers of 6-mercapto-1-hexanol and mercapto-n-hexyl-poly(dT)18-fluorescein on polycrystalline gold surfaces: an electrochemical impedance spectroscopy study," Journal of Electroanalytical Chemistry, vol. 605, no. 2, pp. 145-150, 2007.

[32] W. Cai, J. R. Peck, D. W. van der Weide, and R. J. Hamers, "Direct electrical detection of hybridization at DNA-modified silicon surfaces," Biosensors and Bioelectronics, vol. 19, no. 9, pp. 1013-1019, 2004.

[33] B. C. Jacquot, N. Muñoz, D. W. Branch, and E. C. Kan, "NonFaradaic electrochemical detection of protein interactions by integrated neuromorphic CMOS sensors," Biosensors and Bioelectronics, vol. 23, no. 10, pp. 1503-1511, 2008.

[34] E. Katz and I. Willner, "Probing biomolecular interactions at conductive and semiconductive surfaces by impedance spectroscopy: routes to impedimetric immunosensors, DNAsensors, and enzyme biosensors," Electroanalysis, vol. 15, no. 11, pp. 913-947, 2003.

[35] E. Sharon, R. Freeman, T. V. Ran, and I. Willner, "Impedimetric or ion-sensitive field-effect transistor (ISFET) aptasensors based on the self-assembly of au nanoparticle-functionalized supramolecular aptamer nanostructures," Electroanalysis, vol. 21, no. 11, pp. 1291-1296, 2009.

[36] J. Wang, "Towards genoelectronics: electrochemical biosensing of DNA hybridization," Chemistry-A European Journal, vol. 5, p. 1681, 1999.

[37] S. R. Mikklessen, "Electrochecmical biosensors for DNA sequence detection," Electroanalysis, vol. 8, p. 15, 1996.

[38] E. Paleček and M. Fojta, "Detecting DNA hybridization and damage," Analytical Chemistry, vol. 73, no. 3, 2001.

[39] H. Aoki, P. Buhlmann, and Y. Umezawa, "Electrochemical detection of a one-base mismatch in an oligonucleotide using ion-channel sensors with self-assembled PNA monolayers," Electroanalysis, vol. 12, no. 16, pp. 1272-1276, 2000.

[40] A. J. Bard and L. R. Faulkner, Electrochemical Methods: Fundamentals and Applications, chapter 10, 2nd edition, 2001.

[41] C. Xi, M. Balberg, S. A. Boppart, and L. Raskin, "Use of DNA and peptide nucleic acid molecular beacons for detection and quantification of rRNA in solution and in whole cells," Applied and Environmental Microbiology, vol. 69, no. 9, pp. 5673-5678, 2003.

[42] X. Liu and W. Tan, "A fiber-optic evanescent wave DNA biosensor based on novel molecular beacons," Analytical Chemistry, vol. 71, p. 5054, 1999. 

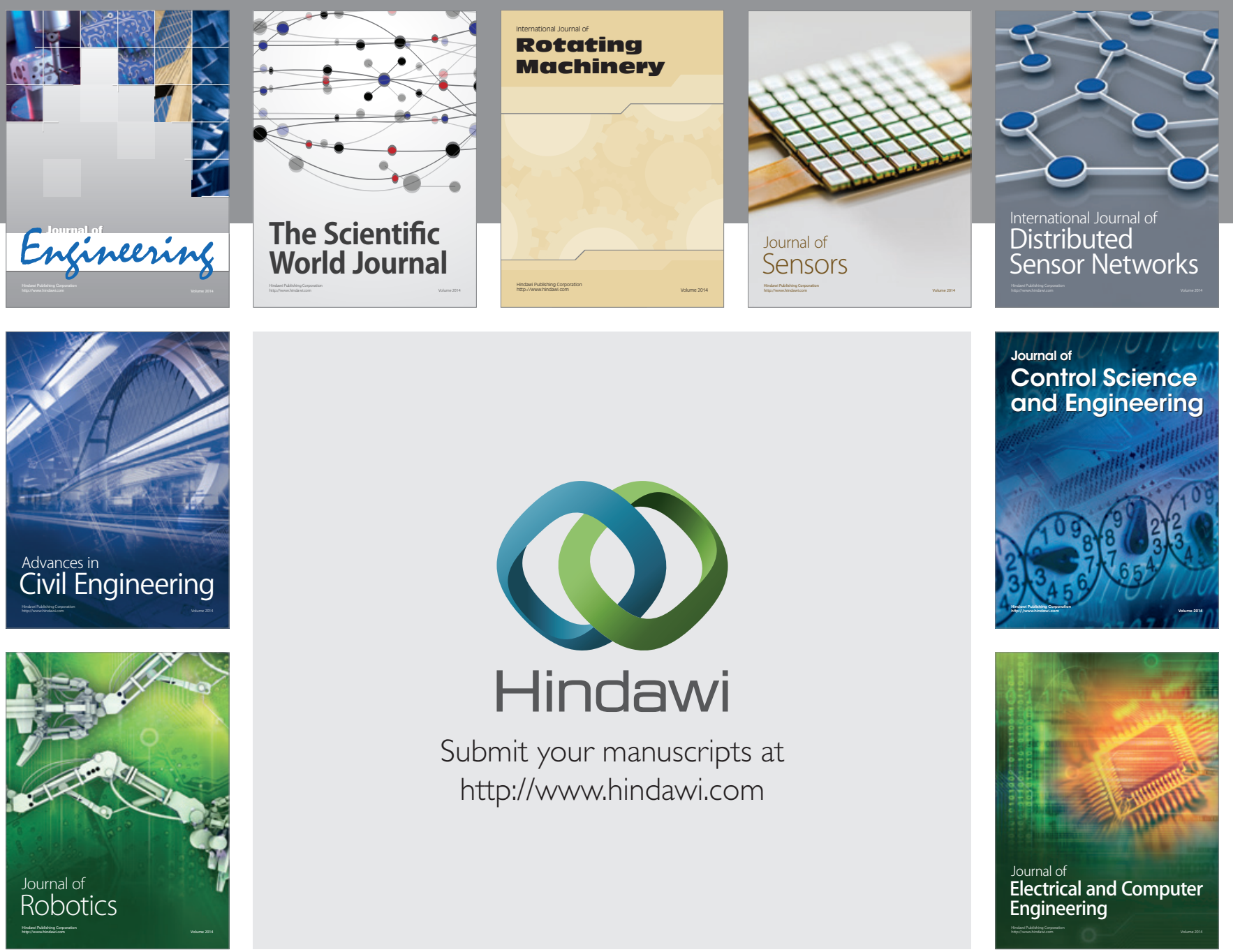

Submit your manuscripts at

http://www.hindawi.com
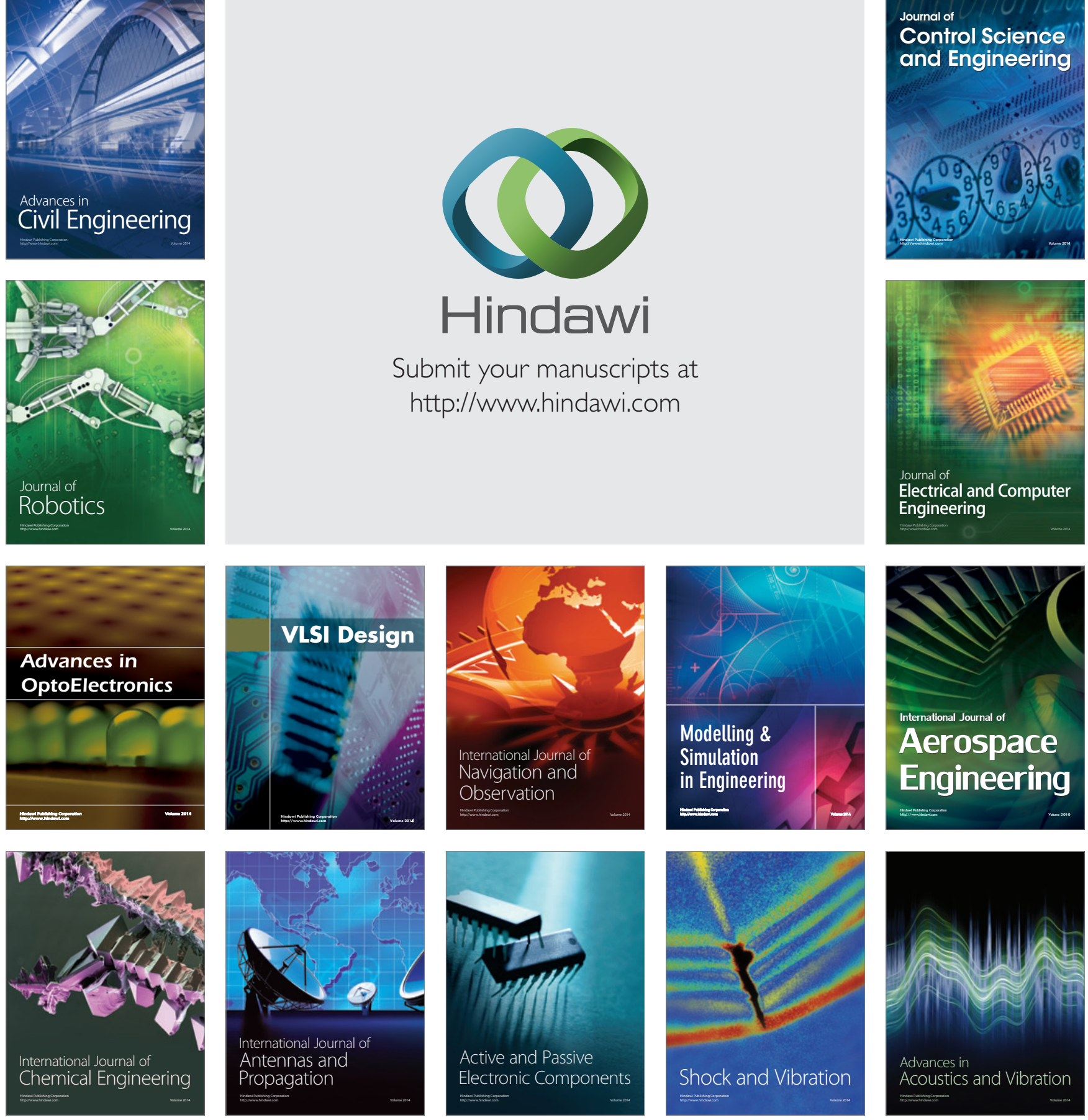